\title{
In vitro Adhesion of Oral Candida dubliniensis Isolates to Acrylic Denture Surfaces following Brief Exposure to Sub-Cidal Concentrations of Polyenes, Azoles and Chlorhexidine
}

\author{
Arjuna N.B. Ellepola ${ }^{\mathrm{a}}$ Bobby K. Joseph ${ }^{\mathrm{a}}$ Yacoub Altarakemah ${ }^{\mathrm{a}}$ \\ Lakshman P. Samaranayake $^{c}$ Sukumaran Anil ${ }^{d}$ Mohamed Hashem ${ }^{e}$ \\ Zia U. Khan ${ }^{\text {b }}$ \\ ${ }^{a}$ Departments of Bioclinical, Diagnostics and Restorative Sciences, Faculty of Dentistry and ${ }^{b}$ Department of \\ Microbiology, Faculty of Medicine, Health Sciences Center, Kuwait University, Jabriya, Kuwait; ' $O$ Oral Microbiomics

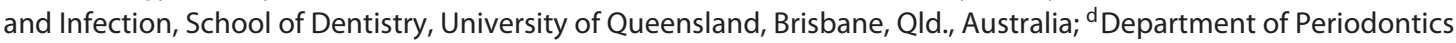

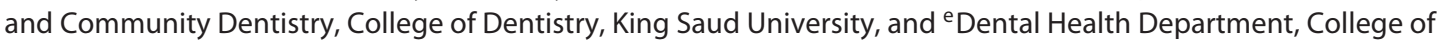 \\ Applied Medical Sciences, King Saud University, Riyadh, Saudi Arabia
}

\author{
Key Words \\ Candida dubliniensis · Nystatin · Amphotericin B • \\ Ketoconazole $\cdot$ Fluconazole $\cdot$ Chlorhexidine . \\ Acrylic dentures
}

\begin{abstract}
Objectives: We aimed to investigate the effect of brief exposure to sub-cidal concentrations of nystatin, amphotericin B, ketoconazole, fluconazole and chlorhexidine gluconate on the adhesion of oral Candida dubliniensis isolates to the surface of acrylic dentures. Methods: After determining the minimum inhibitory concentration of each drug, 20 oral isolates of $C$. dubliniensis were exposed to sub-cidal concentrations of the drugs for $1 \mathrm{~h}$. The drugs were then removed by dilution, and the adhesion of the isolates to denture acrylic strips was assessed by an in vitro adhesion assay. Results: Compared to the controls, exposure to nystatin, amphotericin B, ketoconazole, fluconazole and chlorhexidine gluconate suppressed the ability of $C$. dubliniensis isolates to adhere to acrylic denture surfaces with a reduction of 74.68 ,
\end{abstract}

74.27, 57.31, 44.57 and 56.53\% ( $p<0.001$ for all drugs), respectively. Conclusions: Brief exposure to sub-cidal concentrations of anti-mycotics suppressed the adhesion of C. dubliniensis oral isolates to acrylic denture surfaces.

(C) 2014 S. Karger AG, Basel

\section{Introduction}

Oral candidosis is considered to be the most common human fungal infection, which manifests in a variety of clinical guises. Candida-induced denture stomatitis (CDS) is one form of oral candidosis. Acrylic dentures are an important predisposing factor for CDS as they are usually ill-fitting and, with sub-optimal hygiene, can act as reservoirs of infection [1]. CDS is seen as an inflammatory candidal lesion of the oral mucosa, occurring particularly under complete or partial removable dentures and it affects up to $65 \%$ of denture wearers [2]. High salivary counts of Candida are also much more common in full-denture wearers than in dentate individuals [3]. It is believed that

\begin{tabular}{ll}
\hline KARGER 125:s & $\begin{array}{l}\text { () 2014 S. Karger AG, Basel } \\
1011-7571 / 14 / 0241-0058 \$ 39.50 / 0 \quad \text { Karger }\end{array}$ \\
$\begin{array}{l}\text { E-Mail karger@karger.com } \\
\text { www.karger.com/mpp }\end{array}$ & $\begin{array}{l}\text { This is an Open Access article licensed under the terms of the } \\
\text { Creative Commons Attribution-NonCommercial 3.0 Un- } \\
\text { ported license (CC BY-NC) (www.karger.com/OA-license), } \\
\text { applicable to the online version of the article only. Distribu- } \\
\text { tion permitted for non-commercial purposes only. }\end{array}$
\end{tabular}

Dr. Arjuna Ellepola, BDS, PhD, Associate Prof. in Oral Microbiology

Department of Bioclinical Sciences, Faculty of Dentistry

Kuwait University, PO Box 24923

Safat 13110 (Kuwait)

E-Mail arjuna@hsc.edu.kw 
the ability of Candida sp. to adhere to acrylic surfaces may be important in the pathogenesis of CDS, as adhesion is apparently the initial step in the colonization and subsequent infection of host surfaces by Candida.

Candida dubliniensis is now well-recognized as an opportunistic fungi attendant with recurrent oral candidosis in AIDS patients [4]. It has also been isolated from the oral cavity of diabetic patients and the sputum of cystic fibrosis patients [5]. The fact that C. dubliniensis has been isolated from the upper respiratory tract specimens, from the blood and from a case of endocarditis involving a prosthetic aortic valve suggests that it can disseminate to other sites as well [4, 6-8]. Interestingly, when Candida sp. colonization and CDS were assessed in complete denture wearers in Iran, C. dubliniensis was recovered from 10.9\% of the samples analyzed [9]. C. dubliniensis isolates were also detected in the oral environment of denture wearers in association with C. albicans isolates in 10\% of CDS cases in Brazil [10]. Moreover, when Candida sp. isolated from 50 individuals with CDS were analyzed, C. dubliniensis was recovered from 4 of these individuals [2]. It has thus been suggested that the association between $C$. $d u b$ liniensis and CDS may play an important role in the establishment and persistence of C. dubliniensis-induced CDS [10].

Many therapeutic agents with anti-fungal properties are obtainable for the treatment of CDS $[11,12]$. These agents include polyene anti-fungals such as nystatin and amphotericin B, azoles such as ketoconazole (an imidazole) and fluconazole (a triazole) as well as chlorhexidine gluconate mouthwash $[11,12]$. Despite the availability of these agents for the treatment of CDS, failure of therapy is not uncommon $[11,12]$. For instance, the diluent effect of saliva and the cleansing effect of the oral musculature in the niches of the oral milieu tend to reduce the availability of anti-fungal agents below that of effective therapeutic concentrations, thereby lowering their efficacy. Hence, intra-orally, the pathogenic Candida may undergo a brief exposure to anti-fungal drugs, but after that the drug concentration is likely to become sub-therapeutic $[11,12]$. The ability of C. dubliniensis isolates to adhere to denture acrylic after such brief exposure to anti-fungal drugs has not been studied so far. Based on the above information and the results of a recent study conducted in Kuwait where oral C. dublinienis isolates had the highest prevalence among non-C. albicans oral Candida sp. [13], our objective was to determine the adhesion of oral C. $d u b l i$ niensis isolates to denture acrylic following brief exposure to sub-cidal concentrations of nystatin, amphotericin B, ketoconazole, fluconazole and chlorhexidine gluconate.

\section{Materials and Methods}

\section{Organisms}

We made use of 20 oral isolates of $C$. dubliniensis recovered from the oral-rinse samples of patients attending the Kuwait University Dental Clinic in a previous study [13]. Initially, all the yeast isolates were tested for germ tube (GT) formation. The colony characteristics were then observed using CHROMagar Candida medium (Becton Dickinson \& Co., Sparks, Md., USA), and carbohydrate assimilation profiles were obtained using the VITEK 2 yeast identification system (BioMérieux, Marcy-l'Etoile, France). The identity of C. dubliniensis was confirmed by the production of rough colonies with hyphal fringes and chlamydospores on simplified sunflower seed agar and by using semi-nested PCR amplification of the internally transcribed spacer 2 region of rDNA followed by direct DNA sequencing of the internally transcribed spacer region of rDNA [13].

\section{Anti-Fungal Agents and Media}

Anti-fungal agents were prepared as in previous similar studies $[14,15]$. Briefly, nystatin, amphotericin B, ketoconazole and fluconazole (Sigma, St. Louis, Mo., USA) were dissolved in dimethylsulphoxide. Initially, $10,000 \mu \mathrm{g} / \mathrm{ml}$ of each anti-mycotic agent was prepared and stored at $-20^{\circ} \mathrm{C}$ as previously described $[14,15]$. For $1 \mathrm{~h}$ of exposure to the yeasts, each agent was suspended/diluted in RPMI 1640 medium buffered with 0.165 M MOPS [3-(N-morpholino)propanesulphonic acid] containing L-glutamine and lacking sodium bicarbonate (Sigma), which was dissolved in 1 litre of sterile distilled water and adjusted to a $\mathrm{pH}$ of 7.2 and then filter sterilized. Chlorhexidine gluconate $(0.2 \%$; GlaxoSmithKline, Brentford, UK) was dissolved in sterile phosphate-buffered saline (PBS) at a $\mathrm{pH}$ of 7.2 and diluted to obtain a concentration of $0.005 \%$ prior to each experiment as previously described $[16,17]$.

\section{Determination of Minimum Inhibitory Concentration}

Anti-fungal susceptibility values of $C$. dubliniensis isolates were determined (as in a previous study [13]) against amphotericin B, ketoconazole and fluconazole by Etest and performed according to the manufacturer's recommendations (AB BIODISK, Solna, Sweden). Briefly, each test isolate was freshly sub-cultured. Five isolated colonies were uniformly suspended in sterile saline, and turbidity was adjusted to a $0.5 \mathrm{McF}$ arland standard, which is approximately $1-5 \times 10^{6}$ cells $/ \mathrm{ml}$ according to the 2004 Clinical and Laboratory Standards Institute (CLSI) document M44-A. This inoculum was swabbed onto the agar plates (150 $\mathrm{mm}$ in diameter) and allowed to dry for 10-15 min before the Etest strips were applied. RPMI 1640 agar supplemented with $2 \%$ glucose and buffered with MOPS (0.165 M; pH 7.0) was used for susceptibility testing according to the method recommended by the CLSI document M27-A2. The plates were incubated at $35^{\circ} \mathrm{C}$, and the minimum inhibitory concentration (MIC) values were recorded after $24-48$ $\mathrm{h}$ of incubation. The point where inhibition ellipses intercepted the scale on the anti-fungal strip was taken as the MIC for each test isolate. There was complete inhibition (100\%) of growth for amphotericin B, and a marked decrease in growth intensity $(80 \%)$ for fluconazole and ketoconazole. C. albicans ATCC 90028 and C. parapsilosis ATCC 22019 were the reference strains used for quality control of the susceptibility testing. The interpretive susceptibility break points for fluconazole were those recommended by the CLSI document M27-A2. Due to the lack of defined susceptibility 
break points for amphotericin B and ketoconazole, an isolate was considered susceptible when the MIC break point was $\leq 1.0 \mu \mathrm{g} / \mathrm{ml}$ for amphotericin B and $0.125 \mu \mathrm{g} / \mathrm{ml}$ for ketoconazole [13].

The MIC values of nystatin and chlorhexidine gluconate were determined by the broth dilution technique as done previously [14, 18 ] by performing 2 -fold serial dilutions of the drug in microtitre plates using an inoculum of $1-5 \times 10^{5} \mathrm{CFU} / \mathrm{ml}$. The MIC was determined visually after $24 \mathrm{~h}$ of incubation at $37^{\circ} \mathrm{C}$ [18-20]. It was defined as the lowest concentration of the drug that inhibited the growth of Candida cells, as indicated by the absence of turbidity (i.e. optically clear). It was read independently by 2 laboratory personnel, with C. albicans ATCC 90028 being used as a reference strain.

\section{Preparation of Denture Acrylic Strips}

The acrylic strips for the adhesion assay were prepared as described previously $[1,19]$. In brief, transparent self-polymerizing acrylic powder ( $1.5 \mathrm{~g}$ polymethyl methacrylate powder) was spread on an aluminium foil-covered glass slide $(2.5 \times 7.5 \mathrm{~cm})$. Monomer liquid ( $1 \mathrm{ml}$; DENTSPLY Ltd., Weybridge, UK) was poured on to the surface of the slide, a second slide similar to the first was immediately placed on top of the polymerizing mixture and the slides were then firmly secured at both ends with two binder clips. After bench-curing for $30 \mathrm{~min}$, the glass slides were separated. The resultant acrylic strips were cut into $5 \times 5 \mathrm{~mm}$ squares, immersed in distilled water for 1 week to leach any excess monomer and then washed in running water for $3 \mathrm{~h}$. The strips were disinfected by dipping in $70 \%$ alcohol and then washed with sterile distilled water. They were then ultrasonicated for 20 min to remove any contaminants and artefacts from the surfaces, washed again in sterile distilled water, dried and then used for the adhesion assay.

Preparation of the Cell Suspension for the Adhesion Assay

A previously described method was used [1, 14-19]. Briefly, yeast cells that had been maintained on Sabouraud dextrose agar were inoculated onto fresh plates and incubated overnight at $37^{\circ} \mathrm{C}$ for $24 \mathrm{~h}$ prior to use. The organisms were harvested, and a cell suspension was prepared in sterile PBS at $520 \mathrm{~nm}$ to an optical density of 1.5 . From this cell suspension, $0.5 \mathrm{ml}$ was added to tubes containing $2 \mathrm{ml}$ of RPMI (control) and $2 \mathrm{ml}$ of RPMI/drug (test), in which the sub-cidal drug concentrations were 2 times the MIC values as in previous studies [1,14-19]. This gave a cell suspension of $10^{6}$ cells $/ \mathrm{ml}$ in each assay tube. The tubes were then incubated at $37^{\circ} \mathrm{C}$ for a period of $1 \mathrm{~h}$. Following this limited exposure, the cells were washed twice in PBS and centrifuged for $10 \mathrm{~min}$ at $3,000 \mathrm{~g}$. The supernatant was decanted, and the pellets were resuspended in $6 \mathrm{ml}$ of sterile PBS. This procedure has been used previously for drug removal and has been shown to reduce the concentration of the drug as much as 10,000 times, thereby minimizing any carry-over effects of the drug following its removal [1,14-19]. Viable counts of the control and the test were performed after removal of the drugs. As the procedure of drug removal effectively eliminated any carry-over effect, there was virtually no difference on the viable counts of the control and the tests following exposure to the already-diluted sub-therapeutic drug concentrations as observed in previous studies [1, 14-19].

\section{Adhesion Assay}

A previously applied method was used $[1,19]$. With the help of aseptic techniques, acrylic strips were placed vertically in the wells of a sterile serological plate. Thereafter, $400 \mu \mathrm{l}$ of Candida cell suspension was added to each well, until it completely covered the acrylic strips. The whole assembly was then placed in an incubator for $1 \mathrm{~h}$ at $37^{\circ} \mathrm{C}$ with gentle agitation at $120 \mathrm{rev} / \mathrm{min}$. The strips were then recovered aseptically from the wells and washed 3 times, by dipping gently into sterile PBS which helped to dislodge the loosely attached Candida cells. They were then dried and stained using a modified Gram stain without the counter-stain as done previously. After air-drying at room temperature, they were mounted on glass slides with glycerol, and the adherent Candida was quantified as previously described [20]. Adherent Candida cells in 20 fields of view for each strip $\left(0.25 \mathrm{~mm}^{2} /\right.$ field $)$ were determined using a light microscope at $\times 400$ magnification, and the results were expressed as Candida cells $/ \mathrm{mm}^{2}$. The majority of the attached Candida cells were in the blastospore stage (cells with a rounded format), some with daughter cells and only very few with hyphae or pseudohyphae. The following previously used parameters [20] were used to standardize the counts: a budding yeast cell was considered as a unit cell if the daughter cell was smaller than the mother cell, and a hyphum was counted as a single cell.

All experiments were repeated on three separate occasions with duplicate determinations on each occasion.

\section{Statistical Analysis}

As for previous studies [1, 19], the data obtained from the adhesion assay were analyzed using the ANOVA Dunnet t test, which treats one group as a control (unexposed to drugs) and compares all other groups (exposed to drugs) against this. Variations due to the different drugs on the suppression of adhesion to the denture acrylic were analyzed using the Tukey-Kramer multiple-comparisons tests. A p value of $<0.05$ was considered statistically significant.

\section{Results}

The MIC of the 20 isolates of $C$. dubliniensis to amphotericin $B$ ranged from 0.002 to $0.125 \mu \mathrm{g} / \mathrm{ml}$. For ketoconazole, the range was $0.002-0.012 \mu \mathrm{g} / \mathrm{ml}$. For fluconazole, it was $0.016-0.38 \mu \mathrm{g} / \mathrm{ml}$. The MIC of the $20 \mathrm{C}$. dubliniensis isolates to nystatin ranged from 0.09 to $0.78 \mu \mathrm{g} / \mathrm{ml}$. The MIC of chlorhexidine gluconate for all C. dubliniensis isolates tested was at $0.00125 \%$ dilution.

The candidal adhesion of the unexposed C. dubliniensis isolates to the acrylic denture surfaces was 45.05 cells/ $\mathrm{mm}^{2}$. The adhesion to the denture acrylic following brief exposure to nystatin, amphotericin $\mathrm{B}$, ketoconazole, fluconazole and chlorhexidine gluconate was 11.34, 11.50, $19.14,24.85$ and $19.51 \mathrm{cells} / \mathrm{mm}^{2}$, respectively (table 1 ). Hence, compared to the controls, brief exposure to nystatin, amphotericin B, ketoconazole, fluconazole and chlorhexidine gluconate inhibited the ability of the isolates to adhere to the denture acrylic with a reduction of 74.68\% (range 64.75-82.09\%), 74.27\% (range 63.2183.10\%), 57.31\% (range 47.58-67.40\%), 44.57\% (range
60 
Table 1. Adhesion of C. dubliniensis isolates $\left(\right.$ cells $/ \mathrm{mm}^{2}$ ) to acrylic denture surfaces following $1 \mathrm{~h}$ exposure to and subsequent removal of therapeutic agents with anti-fungal properties

\begin{tabular}{lcrrrrr}
\hline Isolate & Control & \multicolumn{1}{l}{ NYS } & AMP-B & KETO & FLU & CHX \\
\hline CD1 & $48.9 \pm 1.21$ & $11.3 \pm 0.76$ & $11.6 \pm 1.17$ & $18.9 \pm 1.66$ & $23.8 \pm 2.41$ & $19.4 \pm 1.74$ \\
CD2 & $45.3 \pm 1.35$ & $12.9 \pm 0.89$ & $14.1 \pm 2.06$ & $23.4 \pm 1.09$ & $27.1 \pm 2.61$ & $22.8 \pm 1.81$ \\
CD3 & $41.7 \pm 1.78$ & $14.7 \pm 0.79$ & $14.5 \pm 1.08$ & $19.4 \pm 1.73$ & $24.7 \pm 2.23$ & $19.8 \pm 1.49$ \\
CD4 & $42.4 \pm 1.12$ & $14.4 \pm 1.06$ & $15.6 \pm 2.43$ & $21.4 \pm 1.21$ & $26.1 \pm 2.41$ & $20.6 \pm 1.33$ \\
CD5 & $44.8 \pm 1.96$ & $10.6 \pm 2.22$ & $10.3 \pm 1.06$ & $18.3 \pm 2.37$ & $24.8 \pm 1.98$ & $19.1 \pm 1.06$ \\
CD6 & $45.2 \pm 2.31$ & $9.2 \pm 0.93$ & $8.7 \pm 1.14$ & $17.6 \pm 2.33$ & $22.3 \pm 1.84$ & $18.8 \pm 0.93$ \\
CD7 & $47.6 \pm 1.73$ & $11.5 \pm 1.09$ & $12.6 \pm 2.12$ & $21.4 \pm 1.98$ & $27.3 \pm 1.07$ & $22.1 \pm 2.67$ \\
CD8 & $43.5 \pm 2.07$ & $12.6 \pm 1.46$ & $11.8 \pm 0.98$ & $16.7 \pm 0.84$ & $21.9 \pm 2.15$ & $17.8 \pm 1.27$ \\
CD9 & $49.7 \pm 1.46$ & $8.9 \pm 1.84$ & $8.4 \pm 1.03$ & $16.2 \pm 1.76$ & $22.4 \pm 2.33$ & $16.6 \pm 1.83$ \\
CD10 & $48.2 \pm 1.44$ & $12.3 \pm 1.27$ & $11.6 \pm 2.37$ & $16.8 \pm 2.42$ & $20.7 \pm 1.72$ & $16.2 \pm 2.29$ \\
CD11 & $41.4 \pm 1.87$ & $12.6 \pm 1.94$ & $14.4 \pm 2.31$ & $21.7 \pm 2.22$ & $28.9 \pm 1.08$ & $22.1 \pm 2.43$ \\
CD12 & $41.8 \pm 1.91$ & $8.5 \pm 1.08$ & $10.6 \pm 0.88$ & $19.6 \pm 2.38$ & $28.3 \pm 1.23$ & $18.8 \pm 0.92$ \\
CD13 & $46.3 \pm 2.46$ & $11.4 \pm 1.56$ & $11.2 \pm 1.19$ & $20.8 \pm 1.16$ & $28.7 \pm 1.71$ & $21.4 \pm 1.08$ \\
CD14 & $49.7 \pm 2.22$ & $10.1 \pm 2.07$ & $9.3 \pm 1.77$ & $17.8 \pm 1.09$ & $25.1 \pm 2.41$ & $18.4 \pm 0.96$ \\
CD15 & $42.2 \pm 0.93$ & $10.7 \pm 2.11$ & $10.4 \pm 2.42$ & $21.2 \pm 1.84$ & $26.3 \pm 2.19$ & $22.7 \pm 2.07$ \\
CD16 & $41.4 \pm 1.24$ & $10.3 \pm 1.83$ & $9.6 \pm 1.12$ & $18.3 \pm 1.96$ & $23.4 \pm 1.01$ & $17.1 \pm 1.88$ \\
CD17 & $47.9 \pm 2.43$ & $8.7 \pm 1.74$ & $9.8 \pm 2.23$ & $16.7 \pm 2.08$ & $21.7 \pm 1.93$ & $17.8 \pm 1.39$ \\
CD18 & $43.2 \pm 0.84$ & $12.2 \pm 0.86$ & $12.7 \pm 1.04$ & $16.3 \pm 2.01$ & $22.3 \pm 1.26$ & $15.9 \pm 1.76$ \\
CD19 & $48.2 \pm 0.92$ & $14.5 \pm 2.01$ & $12.6 \pm 0.97$ & $24.2 \pm 0.97$ & $30.4 \pm 2.08$ & $26.4 \pm 0.92$ \\
CD20 & $41.6 \pm 1.62$ & $9.4 \pm 0.81$ & $10.2 \pm 1.39$ & $16.1 \pm 1.61$ & $20.8 \pm 1.07$ & $16.4 \pm 2.34$ \\
\hline Mean & 45.05 & 11.34 & 11.50 & 19.14 & 24.85 & 19.51 \\
SEM & 0.68 & 0.43 & 0.46 & 0.55 & 0.66 & 0.61 \\
SD & 3.04 & 1.93 & 2.04 & 2.48 & 2.93 & 2.74 \\
p value & & $<0.001$ & $<0.001$ & $<0.001$ & $<0.001$ & $<0.001$ \\
\hline
\end{tabular}

All values indicate the average of experiments repeated on 3 separate occasions with duplicate determinations on each occasion. AMP-B = Amphotericin B; CHX = chlorhexidine; FLU = fluconazole; $\mathrm{KETO}=$ ketoconazole; NYS = nystatin.

Table 2. The reduction of adhesion of $C$. dubliniensis isolates (cells $/ \mathrm{mm}^{2}$ ) to acrylic denture surfaces following $1 \mathrm{~h}$ exposure to and subsequent removal of therapeutic agents with anti-fungal properties compared to the unexposed controls

\begin{tabular}{lccccc}
\hline & NYS & AMP-B & KETO & FLU & CHX \\
\hline Reduction, $\%$ & 74.68 & 74.27 & 57.31 & 44.57 & 56.53 \\
SEM & 1.09 & 1.21 & 1.41 & 1.72 & 1.46 \\
SD & 4.88 & 5.40 & 6.32 & 7.70 & 6.53 \\
p value & $<0.001$ & $<0.001$ & $<0.001$ & $<0.001$ & $<0.001$ \\
\hline
\end{tabular}

$\mathrm{AMP}-\mathrm{B}=$ Amphotericin $\mathrm{B} ; \mathrm{CHX}=$ chlorhexidine; $\mathrm{FLU}=$ fluconazole; $\mathrm{KETO}=$ ketoconazole; $\mathrm{NYS}=$ nystatin.

$30.19-57.05 \%$ ) and $56.53 \%$ (range $45.23-66.60 \%$ ), respectively (table 2 ). These values were all highly significant $(\mathrm{p}<0.0001)$.

Analysis of the variations between the 5 drugs on the suppression of candidal adhesion to acrylic denture surfaces using the Tukey-Kramer multiple-comparisons tests is shown in table 3. It was revealed that the suppressive effect elicited by nystatin and amphotericin B was significantly higher than and statistically different from that of ketoconazole, fluconazole and chlorhexidine gluconate $(\mathrm{p}<0.001)$. However, the suppressive effects elicited by the 2 polyenes did not differ statistically. The sup- 
Table 3. Statistical analysis with the Tukey-Kramer multiple-comparisons test of the relative efficacy of 5 different therapeutic agents with anti-fungal properties in reducing C. dubliniensis adhesion to acrylic denture surfaces

\begin{tabular}{llllll}
\hline Drug & NYS & AMP-B & KETO & FLU & CHX \\
\hline NYS & - & n.s. & $<0.001$ & $<0.001$ & $<0.001$ \\
AMP-B & n.s. & - & $<0.001$ & $<0.001$ & $<0.001$ \\
KETO & $<0.001$ & $<0.001$ & - & $<0.001$ & n.s. \\
FLU & $<0.001$ & $<0.001$ & $<0.001$ & - & $<0.001$ \\
CHX & $<0.001$ & $<0.001$ & n.s. & $<0.001$ & - \\
\hline
\end{tabular}

The differences in the adhesion-suppressive effect of the drugs were not significant (n.s.) AMP-B = Amphotericin $\mathrm{B}$; $\mathrm{CHX}=$ chlorhexidine; FLU = fluconazole; $\mathrm{KETO}=$ ketoconazole; $\mathrm{NYS}=$ nystatin.

pressive effect elicited by ketoconazole (imidazole) was significantly different from that of the 2 polyenes as well as from fluconazole $(p<0.001)$ but not from chlorhexidine gluconate. The suppressive effect elicited by fluconazole (triazole) was significantly different from that of the 2 polyenes, ketoconazole and chlorhexidine gluconate $(\mathrm{p}<0.001)$.

\section{Discussion}

In this study, the MIC values of the polyene and azole anti-fungal agents for $C$. dubliniensis isolates were within the range of previous studies with the identical C. dubliniensis isolates $[14,15]$. Based on CLSI standard values, all the isolates were susceptible to nystatin, amphotericin $\mathrm{B}$, ketoconazole and fluconazole. An MIC at a dilution of $0.00125 \%$ for chlorhexidine gluconate was observed for C. dubliniensis isolates, which is similar to the MIC value for C. albicans isolates in a previous study for this antiseptic [18].

Our results further indicate that limited exposure of oral C. dubliniensis isolates to nystatin and amphotericin $B$ significantly reduced their ability to adhere to acrylic denture surfaces. Previous studies have also shown that brief exposure of this yeast to these 2 polyene anti-mycotic agents caused significant suppression of adhesion to buccal epithelial cells (BECs), a reduction in germ tube (GT) formation and cell surface hydrophobicity (CSH) $[14,15,21]$. These previous findings, along with the suppression of adhesion of the C. dubliniensis isolates to denture acrylic observed in our study, may be related to the mechanism of action of nystatin and amphotericin B on the Candida cell wall. Polyenes bind avidly to the sterol components in this wall, making it more permeable and leading to the impairment of barrier function, and the leakage of cellular components and metabolic disruption $[11,12,22,23]$. The formation of sterols or their precursors may also be inhibited by the polyenes $[11,12,22,23]$. In addition, the presence of internally collapsed cells with an intact cell wall leaving 'ghost-like' cells and deflated Candida cells after exposure to sub-cidal concentrations of nystatin has been documented [24]. Studies have also demonstrated dynamic changes in the ultra-structural features of the cell wall during morphogenic transformation to GTs, and have shown that the cell wall of GTs possesses stratification comparable to that of the blastospore wall [25]. Therefore, it is reasonable to speculate that polyene-induced changes in the structure of the cell wall of Candida would affect active budding and multiplication, thus suppressing not only its adhesion to denture acrylic as seen in our study, but also its adhesion to BECs, GT formation and CSH following brief exposure to nystatin and amphotericin B.

Limited exposure to the 2 azole drugs, ketoconazole and fluconazole, also significantly reduced C. dubliniensis adhesion to the denture acrylic. Similar suppressive effects on other C. dubliniensis adhesion attributes such as adhesion to BECs, GT formation and CSH following brief exposure to ketoconazole and fluconazole have been reported before [15, 21]. Again, these adhesionrelated suppressive effects may be related to the mode of action of the azoles. These drugs alter the fungal cell membranes by blocking the 14- $\alpha$-demethylation step in the biosynthesis of ergosterol. The consequent depletion of ergosterol and accumulation of 14-a-methyl sterols leads to alterations in a number of membrane-associated functions $[11,12]$. Such alterations could be the reason for the reduced ability to adhere to acrylic denture surfaces that we observed, as well as for the suppressive ef-
Ellepola et al. 
fects on C. dublinienis isolates and other candidal adhesion traits seen in previous studies $[15,21]$ after limited exposure to azoles.

Chlorhexidine gluconate is used as an adjunct in the treatment of oral candidosis. For instance, $0.2 \%$ chlorhexidine gluconate has been used successfully as an oral rinse in the treatment of CDS, while the $2 \%$ suspension is used as an overnight denture disinfectant $[11,12$, 26]. Chlorhexidine gluconate has a dual action on Candida sp.: it is fungicidal at therapeutic concentrations and it significantly suppresses yeast adhesion to both inorganic and organic substrates $[11,12,26]$. Our results also indicated that limited exposure of oral C. dubliniensis isolates to $0.005 \%$ chlorhexidine gluconate could suppress their ability to adhere to denture acrylic. Similar suppressive effects on other C. dubliniensis adhesion attributes such as GT formation and $\mathrm{CSH}$ following brief exposure to sub-therapeutic concentrations of chlorhexidine gluconate have been reported previously $[16,27]$; these effects may be related to the pharmacodynamics of the antiseptic on the cell wall of Candida. Scanning and transmission electron micrographic studies have demonstrated that the anti-fungal effect of this antiseptic is most likely due to a loss of cytoplasmic components, coagulation of nucleoproteins and the associated morphological changes in the structure of the cell wall [28]. Therefore, it is reasonable to speculate that chlorhexidine gluconate, by affecting the cell wall structure, yields an anti-fungal effect and suppresses the ability of C. dubliniensis isolates to adhere to the denture acrylic.

In this study, brief exposure to the polyene anti-fungal agents resulted in a highly significant suppression (approx. 75\%) on adhesion to denture acrylic, compared to that of ketoconazole, fluconazole and chlorhexidine gluconate (approx. 58, 45 and 57\%, respectively). Such differential activity between the polyenes and the azoles may be due to the fundamental dissimilarity in their modes of action. Polyenes are fungicides and azoles are considered to be fungistatic, which may be the reason for the difference in sensitivity of Candida cell surface structures to polyenes. In addition, there was a statistically significant difference between the 2 azoles in terms of their suppressive effect on adhesion to denture acrylic; a low susceptibility was elicited by fluconazole (45\%) compared to ketoconazole (58\%). Although fluconazole is markedly more effective than ketoconazole in the management of candidal infection, its growth inhibitory activity against Candida isolates in vitro is less than that of ketoconazole [11, 12]. Furthermore, there could be subtle differences between the 2 azoles, as ketoconazole is an imidazole and fluconazole is a triazole. It can thus be speculated that these differences may be the reason for the reduced ability of fluconazole to suppress adhesion to denture acrylic as we observed. Notwithstanding this significant in vitro observation, fluconazole is the drug of choice in the management of chronic Candida infections such as those seen in HIV-infected individuals and debilitated patients $[11,12]$. The anti-candidal pharmacodynamics of chlorhexidine gluconate have not yet been fully elucidated, but our results provide a glimpse of its potency, which is still to be fully evaluated.

This study reports the suppression of adhesion of oral C. dubliniensis isolates to denture acrylic by a number of therapeutic agents that possess a wide spectrum of anticandidal pharmacological properties. We included polyenes, imidazole, triazole and chlorhexidine gluconate, which covered a large number of oral C. dubliniensis isolates. Our findings lend further credibility to the use of these anti-fungal agents in the management of CDS. Further investigations, with a larger number of sessile Candida isolates encased in a biofilm as opposed to the planktonic isolates used in this study, are warranted to further augment these findings.

\section{Conclusion}

The findings of this study showed that a brief exposure to sub-therapeutic concentrations of nystatin, amphotericin $\mathrm{B}$, ketoconazole, fluconazole and chlorhexidine gluconate suppressed the adhesion ability of $C$. dubliniensis isolates to denture acrylic in vitro. When used in vivo, there is the possibility that such exposure could reduce the pathogenicity of Candida.

\section{Acknowledgements}

The work was partially supported by Kuwait University Research Grant No. DB 01/13. We thankfully acknowledge the technical advice and support on laboratory procedures in this project provided by Dr. Sumedha Jayathilake (BDS, PhD) of the Oral Microbiology Division, Faculty of Dental Sciences, University of Peradeniya, Sri Lanka and Ms. Rachel Chandy (Faculty of Medicine) and Ms. Preethi John (Faculty of Dentistry) of Health Sciences Center, Kuwait University, Kuwait. 


\section{References}

1 Ellepola AN, Samaranayake LP: Adhesion of oral Candida albicans isolates to denture acrylic following limited exposure to antifungal agents. Arch Oral Biol 1998;43:999-1007.

$>2$ Pereira CA, Toledo BC, Santos CT, et al: Opportunistic microorganisms in individuals with lesions of denture stomatitis. Diagn $\mathrm{Mi}$ crobiol Infect Dis 2013;76:419-424.

-3 Parvien T: Stimulated salivary flow rate, $\mathrm{pH}$ and Lactobacillus and yeast concentration in persons with different types of dentition. Scand J Dent Res 1984;92:412-418.

4 Brandt ME, Harrison LH, Pass M, et al: Candida dubliniensis fungemia: the first four cases in North America. Emerg Infect Dis 2000; 6:46-49.

5 Manfredi M, McCullough MJ, Al-Karaawi $\mathrm{ZM}$, et al: The isolation, identification and molecular analysis of Candida spp. isolated from the oral cavities of patients with diabetes mellitus. Oral Microbiol Immunol 2002;17: 181-185.

$\checkmark 6$ Peltroche-Llacsahuanga $H$, Dohmen $H$, Haase G: Recovery of Candida dubliniensis from sputum of cystic fibrosis patients. Mycoses 2002;45:15-18.

7 Polacheck I, Strahilevitz J, Sullivan DJ, et al: Recovery of Candida dubliniensis from nonhuman immunodeficiency virus-infected patients in Israel. J Clin Microbiol 2000;38:170174.

$>8$ Garcia J, Soch K, Matthew E, et al: Endocarditis caused by Candida dubliniensis. Am J Med Sci 2013;346:237-239.

$>9$ Zomorodian K, Haghighi NN, Rajaee N, et al: Assessment of Candida species colonization and denture-related stomatitis in complete denture wearers. Med Mycol 2011;49:208211.

10 Gasparoto TH, Dionísio TJ, de Oliveira CE, et al: Isolation of Candida dubliniensis from denture wearers. J Med Microbiol 2009;58: 959-962.
11 Ellepola AN, Samaranayake LP: Antimycotic agents in oral candidosis: an overview: treatment of oral candidosis. Dent Update 2000; 27:165-174.

12 Ellepola AN, Samaranayake LP: Oral candidal infections and antimycotics. Crit Rev Oral Biol Med 2000;11:172-198.

13 Ellepola AN, Khan ZU, Joseph BJ, et al: Prevalence of Candida dubliniensis among oral Candida isolates in patients attending the $\mathrm{Ku}$ wait University Dental Clinic. Med Princ Pract 2011;20: 271-276.

14 Ellepola AN, Joseph BK, Chandy R, et al: The postantifungal effect of nystatin and its impact on adhesion attributes of oral Candida dubliniensis isolates. Mycoses 2014;57:56-63.

15 Ellepola AN, Joseph BK, Khan ZU: Changes in germ tube formation and cell-surface hydrophobicity of oral Candida dubliniensis isolates following brief exposure to sub-cidal concentrations of polyene and azole antifungal agents. Mycoses 2013;56:463-470.

16 Ellepola AN, Joseph BK, Khan ZU: Cell surface hydrophobicity of oral Candida dubliniensis isolates following limited exposure to sub-therapeutic concentrations of chlorhexidine gluconate. Mycoses 2013;56:82-88.

17 Ellepola AN: The effect of brief exposure to sub-therapeutic concentrations of chlorhexidine gluconate on germ tube formation of oral Candida dubliniensis. Mycoses 2011; 54:e330-e335.

18 Ellepola AN, Samaranayake LP: In vitro postantifungal effect (PAFE) elicited by chlorhexidine gluconate on oral isolates of Candida albicans. Microb Ecol Health Dis 1999;11: 143-148.
19 Egusa H, Ellepola AN, Nikawa H, et al: Exposure to sub-therapeutic concentrations of polyene antifungals suppresses the adherence of Candida species to denture acrylic. Chemotherapy 2000;46:267-274.

20 Moura JS, da Silva WJ, Pereira T, et al: Influence of acrylic resin polymerization methods and saliva on the adherence of four Candida species. J Prosthet Dent 2006;96:205-211.

21 Ellepola AN, Chandy R, Zia KU: Post-antifungal effect and adhesion to buccal epithelial cells of oral Candida dubliniensis isolates subsequent to limited exposure to amphotericin $\mathrm{B}$, ketoconazole and fluconazole. J Investig Clin Dent 2014, DOI: 10.1111/jicd.12095.

22 Ellepola AN, Samaranayake LP: The in vitro post-antifungal effect of nystatin on Candida species of oral origin. J Oral Pathol Med 1999; 28:112-116.

23 Ellepola AN: Amphotericin B-induced in vitro postantifungal effect on Candida species of oral origin. Med Princ Pract 2012;21:442446.

24 Ellepola AN, Samaranayake LP: The effect of limited exposure to antifungal agents on the germ tube formation of oral Candida albicans. J Oral Pathol Med 1998;27:213-219.

25 Cassone A, Simonetti N, Strippoli V: Ultrastructural changes in the wall during germtube formation from blastospores of Candida albicans. J Gen Microbiol 1973;77:417-426.

26 Ellepola AN, Samaranayake LP: Adjunctive use of chlorhexidine in oral candidoses: a review. Oral Dis 2001;7:11-17.

27 Ellepola AN: The effect of brief exposure to sub-therapeutic concentrations of chlorhexidine gluconate on germ tube formation of oral Candida dubliniensis. Mycoses 2011; 54:e330-e335.

28 Bobichon H, Bouchet P: Action of chlorhexidine on budding Candida albicans: scanning and transmission electron microscopic study. Mycopathologia 1987;100:27-35. 